\title{
Diagnostic accuracy of reflectance confocal microscopy for pigmented skin lesions presenting dermoscopic features of cutaneous melanoma
}

\author{
Katarzyna Podolec ${ }^{1}$, Magdalena Pirowska ${ }^{1}$, Grzegorz Dyduch², Anna Wojas-Pelc ${ }^{1}$
}

'Department of Dermatology, Jagiellonian University Medical College, Krakow, Poland ${ }^{2}$ Department of Pathology, Jagiellonian University Medical College, Krakow, Poland

Adv Dermatol Allergol 2020; XXXVII (4): 531-534 DOI: https://doi.org/10.5114/ada.2019.82742

\begin{abstract}
Introduction: The incidence and mortality of melanoma are rising rapidly. Despite ongoing research and the introduction of new therapeutic methods, advanced melanoma is still considered incurable. Early detection and surgical excision of the tumor increases patients' survival. Since the diagnostic protocol includes surgical excision of all suspicious lesions, it is burdened with a high rate of unnecessary excisions that cause unwanted scarring. This is why the development of accurate diagnostic techniques is crucial. The most common diagnostic tool in early diagnosis of cutaneous melanoma is dermoscopy, though there are emerging new techniques, such as reflectance confocal microscopy and optical coherence tomography.

Aim: To evaluate diagnostic accuracy of reflectance confocal microscopy as a secondary examination in melanocytic lesions previously diagnosed as melanomas by means of dermoscopy.

Material and methods: Forty-six melanocytic lesions presenting dermoscopic features of cutaneous malignant melanoma were examined by means of reflectance confocal microscopy.

Results: The RCM evaluation showed sensitivity at the level of $100 \%$ and specificity at $62 \%$.

Conclusions: It can be estimated that double evaluation of melanocytic lesions by dermoscopy and reflectance confocal microscopy may allow up to $62 \%$ of unnecessary excisions to be avoided.
\end{abstract}

Key words: melanoma, reflectance confocal microscopy, dermoscopy.

\section{Introduction}

According to data collected on melanoma, its incidence has risen and is projected to rise across the world. Estimated annual incidence in Poland has reached level of 2583 cases (cumulative risk 0.45) [1]. Despite emerging new therapies, the key to decrease the mortality rate is still early detection and removal of early-stage tumors, where surgical removal is considered curative [2]. Dermoscopy has been established as a first-line diagnostic tool in a clinical setting, since it has been proven to be more sensitive and specific in comparison to naked-eye evaluation, and it is relatively inexpensive [3]. However, the number of benign pigmented skin lesions unnecessarily excised is still up to $29.4 \%$, depending on the level of the clinician's experience. Digital monitoring utilizing sequential total body photography and videodermoscopy have been proven useful in early, often featureless melanomas. In hard-to-diagnose cases, the use of reflectance confocal microscopy is being proven to increase diagnostic specificity and sensitivity as a second-line examination tool in combination with classical or digital dermoscopy [4-13].

In vivo reflectance confocal microscopy (RCM) is a relatively new optical imaging technique designed to provide high-resolution images of the skin and mucous membranes [14]. The technique relies on low-power near-infrared laser emitting $830 \mathrm{~nm}$ light. Though the basic principles of the technology were described by Minsky in 1957, the technology became accessible for in vivo imaging of human skin in the last decade. Due to near-histological resolution of the acquired images, it is becoming a valuable diagnostic tool in tertiary referral centers. Providing a strong contrast of melanin, the examination makes it possible to visualize melanocytes/ melanophages - RCM is becoming popular in the pre-

Address for correspondence: Katarzyna Podolec MD, PhD, Department of Dermatology, Jagiellonian University Medical College, 8 Skawinska St, 31-066 Krakow, Poland, phone: +48 501543 249, e-mail: kasiapodolec@gmail.com; katarzyna.podolec@uj.edu.pl Received: 21.12.2018, accepted: 20.01.2019. 
histological diagnosis of melanoma [10, 14-16]. For the examination, a metal ring with a translucent template is placed on the surface of the skin lesion and fixed with special adhesive tape to stabilize the imaging. The ring is then magnetically attached to the objective lens housing. The obtained images are $500 \times 500 \mu \mathrm{m}$ large and may be acquired as a stack of horizontal pictures (VivaStack) or may be arranged in a larger horizontal mosaic (VivaBlock) montaged into horizontal images sized up to $8 \times 8 \mathrm{~mm}$ [17].

\section{Aim}

The aim of the study was to evaluate diagnostic accuracy of reflectance confocal microscopy as a secondary examination in melanocytic lesions previously diagnosed as melanomas by means of dermoscopy.

\section{Material and methods}

A total of 46 melanocytic lesions presenting dermoscopic features of cutaneous malignant melanoma were examined by means of reflectance confocal microscopy at the Department of Dermatology, Jagiellonian University Medical College between 2015 and 2017.

Atypical lesions were excised on the day of examination or within 2-5 days after the imaging. All of the excised skin tumors were evaluated by a pathologist with histopathology examination standardized for cutaneous melanoma. The digital data (both videodermoscopy and RCM images) recorded before the excisions are stored at the Dermatology Department, Jagiellonian University Medical College in Krakow.

All consecutive cases were skin lesions presenting dermoscopic criteria of malignant melanoma according to a 3-point checklist as well as a 7-point checklist (score $\geq 3$ ). Skin lesions were evaluated by means of immersion-based and polarized handheld dermoscopes (Heine Delta 20/Heine Delta 20 Plus; Heine Optotechnik). Dermoscopic images were acquired by means of videodermoscopes (Fotofinder, TeachScreen GmbH, Bad Birnbach, Germany) using minimum 20× magnification.

Reflectance confocal microscopy images were acquired with a Vivascope 1500 (MAVIG GmbH, Munich, Germany), which employs an 830-nm laser beam with a maximum power of $20 \mathrm{~mW}$. In every lesion, 3 horizontal mosaics (VivaBlock; $8 \times 8 \mathrm{~mm}$ ) were recorded at a selected depth representing the epidermis (stratum granulosum/spinosum), dermo-epidermal junction (DEJ) and superficial dermis. Additionally VivaStacks - with a field of view of approximately $500 \times 500 \mu \mathrm{m}$, special resolution of $1.0 \mu \mathrm{m}$ in the lateral dimension and $4-5 \mu \mathrm{m}$ in the axial dimension - were recorded in portions of melanocytic lesions representing the most atypical dermoscopic features. In larger lesions, due to the limited field of vision in reflectance confocal microscope $(8 \times 8 \mathrm{~mm}$ per 1 VivaBlock), the RCM imaging centered on the most dermoscopically challenging parts of tumors, where multiple VivaBlocks were recorded and considered 1 examination. Instrument and acquisition procedures are described elsewhere [14]. One of the atypical pigmented lesions was excluded from the study due to the low quality of RCM images caused by the uneven surface limiting the ability to acquire the image.

The RCM evaluation was based on diagnostic criteria first described by Pellacani et al. Cell atypia at the level of the dermo-epidermal junction and non-edged papillae were considered major criteria. Roundish pagetoid cells, widespread pagetoid infiltration, cerebriform nests and nucleated cells within the upper dermis were considered minor criteria. The features were evaluated for presence/ absence (non-parametric binary data). Tumors presenting 2 major criteria or 1 major and 2 minor criteria were considered malignant [18].

Two dermatologists with a minimum of 6 years' experience conducted an evaluation of both dermoscopic and RCM images. Since the new ordinance of the Ministry of Health requires 2 pathologists to evaluate malignancies, it was decided that 2 dermatologists would evaluate every lesion to confirm the diagnosis. For the purpose of assessment, all images were anonymized to ensure that the physicians were blinded to the clinical diagnosis. For blinding reasons, RCM images were assessed separately - prior to the evaluation of videodermoscopic images (which was conducted to confirm the diagnostic criteria of melanoma). The examination was also blinded from histology results for both dermatologists.

The evaluated group consisted of 46 patients who came to the Dermatology Department with suspicious lesions. The group comprised 25 (4.35\%) male and $21(45.65 \%)$ female patients. The patients' age ranged between 26 and 87 with the average age of 59.9 .

\section{Results}

Histology revealed a total of 33 melanomas per 46 (72\%) lesions and 13 (26.67\%) non-melanoma melanocytic lesions. In RCM 38 (79\%) lesions were interpreted as malignant and 8 (17\%) were evaluated as benign/ dysplastic melanocytic nevi. Melanoma diagnosis based on reflectance confocal microscopy was made in all 33 pathology-positive cases. That means that the sensitivity of melanoma diagnosis in our study reached 100\%. Five benign/dysplastic melanocytic lesions were misdiagnosed as melanomas by means of RCM. The specificity of reflectance-mode confocal microscopy in the study was $62 \%(p<0.001)$.

The evaluated RCM features of both malignant and benign (in histology) lesions and their frequency are summarized in Table 1. In over half of the lesions, cell atypia at the dermo-epidermal junction (80\%), non-edged papil- 
lae (74\%), roundish pagetoid cells (52\%) and widespread pagetoid infiltration (67\%) were observed.

By means of multivariate regression analysis, the following features were associated with positive (confirmed in pathology) RCM melanoma diagnosis: cell atypia in dermo-epidermal junction ( $\mathrm{OR}=21.7, p<0.001$ ) present in 31 of 33 cases; non-edged papillae $(O R=5.60$; $p<0.022$ ) present in 28 of 33 cases; widespread pagetoid infiltration $(\mathrm{OR}=28.0, p<0.001)$ present in $28(84.8 \%)$ of 33 cases; cerebriform nests $(p<0.001)$ present in 3 of 33 cases. The highest sensitivity score was related to cell atypia in the DEJ (93.9\%; $p<0.001)$ and the highest specificity was calculated for cerebriform nests (100\%; $p<0.001$ ) (Table 2).

\section{Discussion}

Dermoscopy has been described as a valuable, inexpensive method for the diagnosis of cutaneous melanoma in routine dermatology practice. The non-invasive microscopic evaluation could create the opportunity to reduce the number of surgical excisions of benign nevi by providing more accurate pre-histological examination.

In our study, we selected 46 melanocytic lesions suspicious for malignant melanoma. Most researchers evaluate the use of RCM in dermoscopically equivocal lesions. In our study, we evaluated only the skin lesions with a dermoscopic image fulfilling the criteria of cutaneous malignant melanoma. Due to high dermoscopy scores, and hence clinical suspicion of malignancy, all lesions were excised. In order to increase the diagnostic accuracy, reflectance confocal microscopy examinations were performed.

In this study, we obtained 100\% sensitivity for melanoma diagnosis with RCM, which is considered high in comparison to other studies showing sensitivity at the level of > 90\% up to 100\% (if RCM is used together with dermoscopy) [5-7, 9].

The RCM specificity in our study was at the level of $62 \%$. The result was lower than in studies conducted by Pellacani et al. (80.21\%) [5], as well as Alarcon et al. (92.4\%) [6] and Curchin et al. (75\%) [7]. Specificity of reflectance confocal microscopy in previously conducted
Table 1. Frequency of reflectance confocal microscopy (RCM) features in both malignant and benign (in histology) lesions

\begin{tabular}{lccc}
\hline RCM & $\boldsymbol{N}$ & $\%$ & Total \\
\hline Cell atypia at DEJ & 37 & 80 & 46 \\
\hline Non-edged papillae & 34 & 74 & 46 \\
\hline Roundish pagetoid cells & 24 & 52 & 46 \\
\hline Widespread pagetoid infiltration & 31 & 67 & 46 \\
\hline Cerebriform nests & 3 & 7 & 46 \\
\hline Nucleated cells within upper dermis & 21 & 46 & 46 \\
\hline
\end{tabular}

studies was mostly researched for RCM used together with dermoscopy - as a 2-step algorithm. In our study, dermatologists evaluating confocal images were blinded to the dermoscopy images prior to RCM evaluation, so that dermoscopy would not influence RCM evaluation. However, after the results were collected, both researchers reevaluated the dermoscopic images in order to confirm the dermoscopic diagnosis of melanoma.

Considering RCM features evaluated in the study, the highest sensitivity score was related to cell atypia in the DEJ $(93.9 \% ; p<0.001)$ and the highest specificity was calculated for cerebriform nests $(100 \% ; p<0.001)$.

Considering the clinical and dermoscopic diagnosis of melanoma, it is certainly useful to reevaluate tumors with reflectance confocal microscopy. By improving the accuracy of examination, RCM can increase the confidence of in vivo examination, and due to an increase of specificity of melanoma detection, it can decrease the number of unnecessary excisions. The technique can free the patients of unnecessary scarring and decrease the cost of diagnosis [19]. It can be estimated that double evaluation of melanocytic lesions by dermoscopy and reflectance confocal microscopy may allow up to $62 \%$ of unnecessary excisions to be avoided. In order to achieve the best possible diagnostic outcome, we think that the RCM specialist should have access to a clinical and dermoscopic image together with RCM [20].

Like all other techniques, RCM also has its limitations. The microscopes have limited image acquisition ability

Table 2. Multivariate logistic regression analysis for reflectance confocal microscopy (RCM) features as predictors

\begin{tabular}{|c|c|c|c|c|c|c|}
\hline Predictor & $\begin{array}{l}\text { Predictor absent } \\
\qquad N(\%)\end{array}$ & $\begin{array}{l}\text { Predictor present } \\
\qquad N(\%)\end{array}$ & $P$-value & OR $(95 \% \mathrm{Cl})$ & Sensitivity & Specificity \\
\hline Cell atypia at DEJ & $2(22.2)$ & $31(86.1)$ & 0.001 & $21.70(3.47-135.75)$ & 93.9 & 58.3 \\
\hline Non-edged papillae & $5(45.5)$ & $28(82.4)$ & 0.022 & $5.60(1.28-24.56)$ & 84.8 & 50.0 \\
\hline Roundish pagetoid cells & $16(72.7)$ & $17(73.9)$ & 0.928 & $1.06(0.28-3.98)$ & - & - \\
\hline Widespread pagetoid infiltration & $5(33.3)$ & $28(93.3)$ & $<0.001$ & $28.00(4.67-168.00)$ & 84.8 & 83.3 \\
\hline Cerebriform nests & $30(71.4)$ & $3(100.0)$ & $<0.001$ & - & 9.1 & 100.0 \\
\hline $\begin{array}{l}\text { Nucleated cells within upper } \\
\text { dermis }\end{array}$ & $15(62.5)$ & $18(85.7)$ & 0.089 & $3.60(0.82-15.74)$ & & \\
\hline
\end{tabular}


in uneven surfaces (e.g., ulceration) and limited depth of examination. It also requires extensive training from a dermatologist.

\section{Conflict of interest}

The authors declare no conflict of interest.

\section{References}

1. Matthews NH, Li WQ, Qureshi AA, et al. Chapter 1: Epidemiology of melanoma. In: Cutaneous Melanoma: Etiology and Therapy [Internet]. doi:10.15586/codon.cutaneousmelanoma.2017.ch1.

2. Rutkowski P, Wysocki PJ, Nasierowska-Guttmejer A, et al. Cutaneous melanoma - guidelines for diagnostics and therapy in 2016. Dermatology Rev 2016; 103: 1-18.

3. Bafounta ML, Beauchet A, Aegerter P, Saiag P. Is dermoscopy (epiluminescence microscopy) useful for the diagnosis of melanoma? Results of a meta-analysis using techniques adapted to the evaluation of diagnostic tests. Arch Dermatol 2001; 137: 1343-50.

4. Chen LL, Scope A, De Carvalho N, et al. Difficult-to-diagnose facial melanomas: utility of reflectance confocal microscopy in uncovering the diagnosis. JAAD Case Rep 2017; 3: 379-83.

5. Pellacani G, Pepe P, Casari A, Longo C. Reflectance confocal microscopy as a second-level examination in skin oncology improves diagnostic accuracy and saves unnecessary excisions: a longitudinal prospective study. Br I Dermatol 2014; 171: 1044-51.

6. Alarcon I, Carrera C, Palou J, et al. Impact of in vivo reflectance confocal microscopy on the number needed to treat melanoma in doubtful lesions. Br J Dermatol 2014; 170: 802-8.

7. Curchin CES, Wurm EMT, Lambie DL, et al. First experiences using reflectance confocal microscopy on equivocal skin lesions in Queensland. Australas J Dermatol 2011; 52: 89-97.

8. Agozzino M, Ferrari A, Cota C, et al. Reflectance confocal microscopy analysis of equivocal melanocytic lesions with severe regression. Skin Res Technol 2018; 24: 9-15.

9. Ferrari B, Pupelli G, Farnetani F, et al. Dermoscopic difficult lesions: an objective evaluation of reflectance confocal microscopy impact for accurate diagnosis. J Eur Acad Dermatology Venereol 2015; 29: 1135-40.

10. Maher NG, Solinas A, Scolyer RA, et al. Detection of desmoplastic melanoma with dermoscopy and reflectance confocal microscopy. J Eur Acad Dermatology Venereol 2017; 31: 2016-24.

11. Edwards SJ, Osei-Assibey G, Patalay R, et al. Diagnostic accuracy of reflectance confocal microscopy using VivaScope for detecting and monitoring skin lesions: a systematic review. Clin Exp Dermatol 2017; 42: 266-75.

12. Urvanegia AC, Tavoloni Braga JC, Shitara D, et al. Reflectance confocal microscopy features of BRAF V600E mutated thin melanomas detected by immunohistochemistry. PLoS One 2017; 12: e0179745.

13. Laimer M, Arzberger E, Kirchner C, et al. Noninvasive Rcm for differentiation of melanotic macules from melanocytic lesions-blinded evaluation of a series of 42 pigmented macules. Dermatol Surg 2017; 43: 911-9.

14. Rajadhyaksha M, Grossman M, Esterowitz D, et al. In vivo confocal scanning laser microscopy of human skin: melanin provides strong contrast. J Invest Dermatol 1995; 104: 946-52.
15. Uribe P, Collgros H, Scolyer RA, et al. In vivo reflectance confocal microscopy for the diagnosis of melanoma and melanotic macules of the lip. JAMA Dermatol 2017; 153: 882-91.

16. Maher NG, Solinas A, Scolyer RA, et al. Detection of desmoplastic melanoma with dermoscopy and reflectance confocal microscopy. J Eur Acad Dermatology Venereol 2017; 31: 2016-24.

17. Kose K, Gou M, Yélamos O, et al. Automated video-mosaicking approach for confocal microscopic imaging in vivo: an approach to address challenges in imaging living tissue and extend field of view. Sci Rep 2017; 7: 10759.

18. Pellacani G, Cesinaro AM, Seidenari S. Reflectance-mode confocal microscopy of pigmented skin lesions - improvement in melanoma diagnostic specificity. J Am Acad Dermatol 2005; 53: 979-85.

19. Serban ED, Farnetani F, Pellacani G, Constantin MM. Role of in vivo reflectance confocal microscopy in the analysis of melanocytic lesions. Acta Dermatovenerol Croat 2018; 26: 64-7.

20. Yélamos O, Nehal KS. Integrating clinical information, dermoscopy and reflectance confocal microscopy to improve the diagnostic accuracy and confidence of amelanotic and lightly pigmented melanomas. Br J Dermatol 2016; 175: 1147-8. 